INGENIERÍA QUÍMICA

\title{
Mineralización fotocatalítica de agua residual contaminada con dicloxacilina comercial en un reactor solar CPC a escala piloto
}

\section{Photocatalytic mineralization of wastewater polluted with commercial dicloxacillin in a pilot-scale solar CPC reactor}

\author{
José Á. Colina-Márquez*\$, Deyler R. Castilla-Caballero* \\ * Programa de Ingeniería Química, Universidad de Cartagena, Cartagena, Colombia. \\ §jcolinam@unicartagena.edu.co,draf.castilla@gmail.com
}

(Recibido: Octubre 04 de 2012 - Aceptado: Abril 02 de 2013)

\begin{abstract}
Resumen
En el presente trabajo se estudió la mineralización de una muestra de agua residual contaminada con dicloxacilina comercial mediante fotocatálisis solar heterogénea con $\mathrm{TiO}_{2}$. Los ensayos se llevaron a cabo en un reactor tipo colector parabólico compuesto (CPC) ubicado el Laboratorio de Fotocatálisis Solar de la Universidad del Valle (Cali, Colombia). Se empleó un diseño experimental de Taguchi para analizar los efectos del pH inicial de la solución y la concentración del catalizador en la mineralización fotocatalítica de la muestra, considerando la energía UV acumulada como variable de ruido. Los resultados mostraron que se alcanzaron reducciones de carbono orgánico total (COT) de hasta $45 \%$. De acuerdo con los valores de señal/ruido (S/R) obtenidos a partir del diseño de Taguchi, la mineralización se favoreció con un $\mathrm{pH}$ inicial de 5 y una concentración de catalizador de $0.3 \mathrm{~g} / \mathrm{l}$.
\end{abstract}

Palabras clave: Dicloxacilina, fotocatálisis heterogénea, $\mathrm{Ti}_{2}, \mathrm{CPC}, \mathrm{pH}$.

\begin{abstract}
In this work, the mineralization of a wastewater sample polluted with commercial dicloxacillin by $\mathrm{TiO}_{2}$-based solar heterogeneous photocatalysis was studied. The experimental tests were carried out in a compound parabolic collector (CPC) type reactor located at the Solar Photocatalytic Laboratory of the Universidad del Valle (Cali, Colombia). A Taguchi experimental design was used for evaluating the effects of the initial $\mathrm{pH}$ of the solution and the catalyst concentration on the photocatalytic mineralization of the polluted sample. The results exhibited that total organic carbon (TOC) removal up to $45 \%$ could be achieved by $\mathrm{TiO}_{2}$-based solar photocatalysis. According to the signal-to-noise ratios obtained from the Taguchi experimental design, the wastewater mineralization was favored at initial $\mathrm{pH}$ of 5 and $\mathrm{TiO}_{2}$ concentration of $0.3 \mathrm{~g} / \mathrm{l}$.
\end{abstract}

Keywords: Dicloxacillin, heterogeneous photocatalysis, $\mathrm{TiO}_{2}, \mathrm{CPC}, \mathrm{pH}$. 


\section{Introducción}

La dicloxacilina (Figura 1) es una isoxazolilpenicilina utilizada en el tratamiento de las infecciones producidas por los estafilococos resistentes a las bencil-penicilinas (Raj et al., 2007). Además de su uso en personas, también se emplea ampliamente en la medicina veterinaria. Se ha reportado que el $65 \%$ de la dicloxacilina que entra al cuerpo humano, se excreta vía urinaria y fecal en su forma química original (Khan \& Ongerth, 2004; Hirsch et al., 1999). Debido a su considerable solubilidad en agua y baja biodegradabilidad (Jjemba 2006; Khan \& Ongerth, 2004), gran parte de este fármaco $\mathrm{y}$ sus metabolitos permanecen intactos al pasar por plantas convencionales de tratamiento de aguas, lo que representa una amenaza para los cuerpos de agua naturales que posteriormente los reciben. Cuando entran al medio ambiente pueden aumentar la resistencia de ciertos microorganismos patógenos a los tratamientos con antibióticos, lo que ocasionaría graves problemas de salud pública. Debido a esto, se hace necesario desarrollar tecnologías especiales para tratar adecuadamente este contaminante refractario.

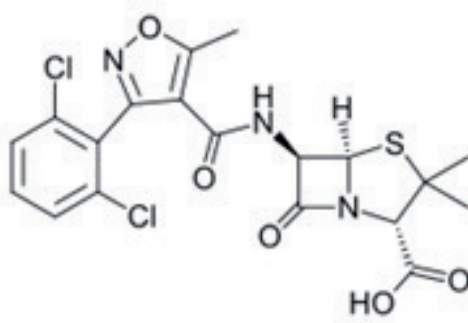

Figura 1. Estructura química de la dicloxacilina.

En las últimas décadas, los procesos avanzados de oxidación (PAOs) han probado ser muy efectivos en la eliminación de efluentes recalcitrantes líquidos y gaseosos (Andreozzi et al., 2005; Yang et al., 2007; Kavitha \& Palanivelu, 2005; Zhong et al., 2007; Klamerth et al., 2009). Se basan en la generación de radicales $\mathrm{OH} \bullet$, los cuales poseen un alto potencial oxidante. Entre los PAOs, la fotocatálisis heterogénea con $\mathrm{TiO}_{2}$ se ha mostrado como una tecnología promisoria y viable para el tratamiento de aguas residuales con contaminantes emergentes. Entre sus ventajas se encuentran la ausencia de limitaciones por transferencia de masa en el proceso y fácil adaptabilidad a las condiciones ambientales; por otra parte, el $\mathrm{TiO}_{2}$ está disponible comercialmente, es de bajo costo, fotoquímicamente estable y no es tóxico (Elmolla \& Chaudhuri, 2010). El mecanismo general de degradación fotocatalítica con dióxido de titanio ha sido ampliamente estudiado (Elmolla \& Chaudhuri, 2010; Kamble et al., 2006; Konstantinou \& Albanis, 2003) y se muestra en las Ec. 1-7. Cuando el $\mathrm{TiO}_{2}$ es irradiado con fotones con energía mayor que su band-gap ( $\left.\mathrm{hv}>\mathrm{E}_{\mathrm{bg}}=3.2 \mathrm{eV}\right)$, los electrones $\left(\mathrm{e}^{-}\right)$ de la banda de valencia (bv) son expulsados a la banda de conducción (bc), formándose huecos $\left(\mathrm{h}^{+}\right)$en la banda de valencia (Ec. 1). En el caso del $\mathrm{TiO}_{2}$, la longitud de onda de los fotones debe ser menor que $387 \mathrm{~nm}$, los cuales se pueden obtener en el rango de la radiación UV. Por otra parte, los huecos generados interactúan con moléculas de agua o iones $\mathrm{OH}^{-}$adsorbidos en la superficie del catalizador, para producir radicales $\mathrm{OH} \cdot$ (Ec. 2-3). La degradación de la materia orgánica se lleva a cabo principalmente mediante la reacción entre la sustancia con los radicales hidroxilos (Ec. 4), aunque también (y menos importante) través del ataque directo de los huecos (Ec. 5). Por su parte, los electrones expulsados, pueden reducir cierto tipo de aceptores de electrones, tales como oxígeno adsorbido o disuelto en el agua, formando aniones superóxido (Ec. 6). Esta etapa es muy importante, debido a que evita la recombinación del par electrón - hueco (Ec. 7) y la disipación la energía radiante en forma de calor.

$$
\begin{gathered}
\mathrm{TiO}_{2}+h v \rightarrow e_{\mathrm{bc}}^{-}+h^{+}{ }_{\mathrm{bv}} \\
h_{\mathrm{vb}}^{+}+\mathrm{H}_{2} \mathrm{O} \rightarrow \mathrm{H}^{+}+\mathrm{OH} . \\
h^{+}{ }_{\mathrm{vb}}+\mathrm{OH}^{-} \rightarrow \mathrm{OH} .
\end{gathered}
$$

Sustancia orgánica $+\mathrm{OH} \cdot \rightarrow$ Productos de la degradación

Sustancia orgánica $+h_{\mathrm{vb}}^{+} \rightarrow$ Productos de la degradación 


$$
\begin{gathered}
e^{-}{ }_{\mathrm{cb}}+\mathrm{O}_{2} \rightarrow \mathrm{O}_{2}^{\cdot^{-}} \\
e_{\mathrm{bc}}^{-}+h_{\mathrm{bv}}^{+} \rightarrow \mathrm{TiO}_{2}
\end{gathered}
$$

La fotocatálisis heterogénea con $\mathrm{TiO}_{2}$ ha sido ampliamente utilizada en la degradación de pesticidas, tintas, fungicidas, fármacos $\mathrm{y}$ otros compuestos refractarios (Colina et al., 2009; Mascolo et al., 2007; Danion et al., 2006; Méndez et al., 2009). Recientemente, se ha empleado en el tratamiento de penicilinas sintéticas tales como amoxicilina, ampicilina y cloxacilina, en las cuales hasta se ha logrado la mineralización completa de las mismas (Elmolla \& Chaudhuri, 2010; Klauson et al., 2010; Rizzo et al., 2009). Sin embargo, se desconoce el efecto de los procesos avanzados de oxidación $\mathrm{y}$, por ende, fotocatálisis heterogénea con $\mathrm{TiO}_{2}$ sobre efluentes con dicloxacilina.

En el presente trabajo se evaluó la mineralización fotocatalítica a escala piloto de una muestra de agua residual sintética contaminada con dicloxacilina comercial, empleando $\mathrm{TiO}_{2}$ como catalizador y la radiación solar como fuente de fotones. Las pruebas se llevaron a cabo en un reactor solar
CPC (colector parabólico compuesto), debido a que ha sido reportado como el más eficiente en la degradación de efluentes orgánicos a escala piloto (Colina, et al., 2009; Bandala, et al., 2004). El reactor permite un mejor aprovechamiento de la radiación solar difusa y directa, a través de los reflectores en forma de involuta que lo conforman (Figura 2). Por otro lado, se evaluó el efecto del $\mathrm{pH}$ inicial de las muestras y la concentración del catalizador $\mathrm{TiO}_{2}$ usando un diseño experimental de Taguchi, y considerando la energía UV acumulada debido a la variación de la intensidad de la radiación solar UV a lo largo del día (Chuen et al., 2009).

\section{Metodología}

\subsection{Reactivos y sustancias químicas}

Las muestras de agua residual sintética se prepararon con dicloxacilina usando cápsulas de la marca comercial Dicloxacilina La Santé ${ }^{\circledR}$. Se utilizó el $\mathrm{TiO}_{2}$ Evonik Degussa P-25 (80\% Anatasa-20\% Rutilo) como catalizador, el cual posee excelentes propiedades como semiconductor (Amhed, et al., 2011). El pH

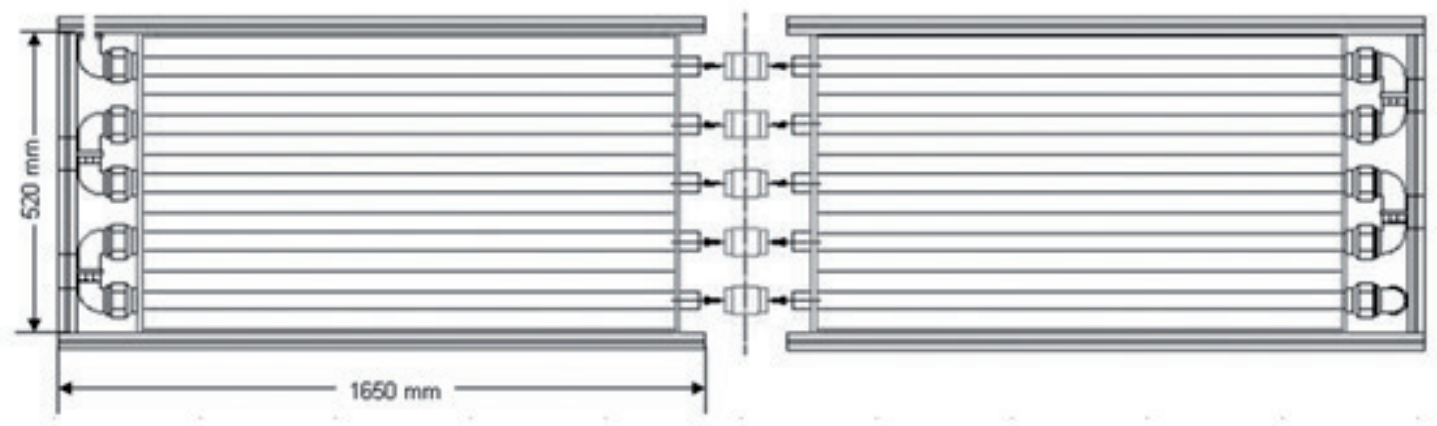

(a)

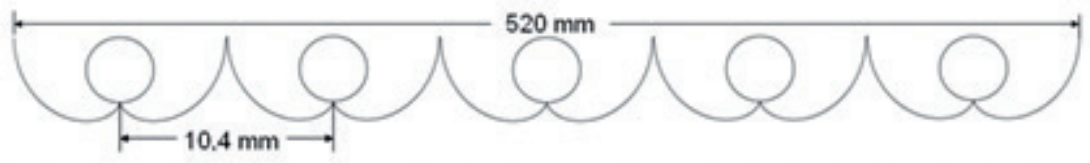

(b)

Figura 2. Reactor CPC: (a) Esquema general, (b) Tubos y reflectores (vista transversal) 
se ajustó con soluciones $1 \mathrm{~N}$ de $\mathrm{HCl}$ y $\mathrm{NaOH}$. En todos los experimentos se empleó agua del grifo para simular un efluente contaminado con dicloxacilina comercial.

\subsection{Procedimiento}

Las soluciones del fármaco se prepararon diluyendo cápsulas de $500 \mathrm{mg}$ de Dicloxacilina La Santé ${ }^{\circledR}$ en 20 litros de agua, para obtener una composición de 25 ppm. Para los experimentos, el pH incial se ajustó en 5 y 9 , y la concentración de $\mathrm{TiO}_{2}$ se fijó en 0.3 y $0.6 \mathrm{~g} / 1$, de acuerdo a trabajos similares realizados en fármacos (Klavaroti et al., 2009; Andreozzi et al., 2004). Los ensayos experimentales se realizaron en el reactor fotocatalítico solar a escala piloto CPC del Laboratorio de Fotocatálisis Solar de la Universidad del Valle (Cali, Colombia) (Figura 3). El reactor, que opera en régimen semi-batch, está conformado por 10 tubos de vidrio SCHOTT Duran ${ }^{\circledR}$ de $32 \mathrm{~mm}$ de diámetro, $1.4 \mathrm{~mm}$ de espesor y $1.2 \mathrm{~m}$ de largo, dispuestos en 5 hileras, colocados sobre reflectores en forma de involuta. Este tipo de reflectores permite aprovechar tanto la componente directa como difusa de la radiación solar (Colina et al., 2009). En la Figura 2 se ilustra el esquema general del reactor $\mathrm{CPC}$ y una vista transversal de los tubos y colectores que lo conforman. Adicionalmente, consta de un tanque de recirculación - alimentación de 20 litros, en el cual, la suspensión con el contaminante se satura de oxígeno del aire mezclado con la corriente líquida de retorno.

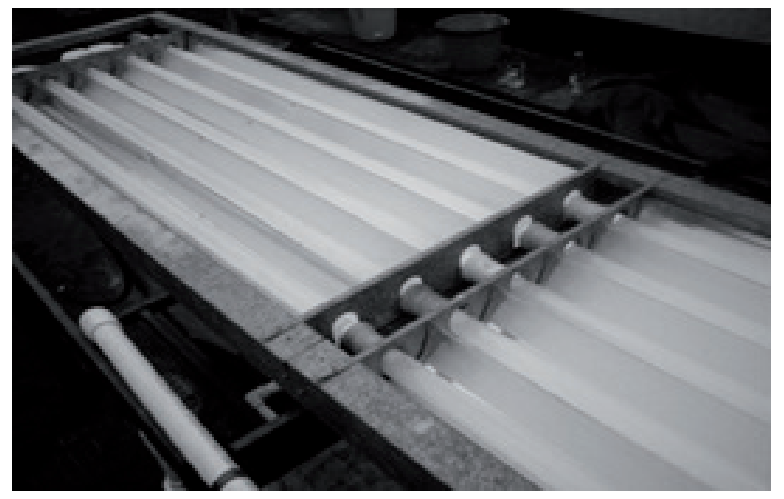

Figura 3. Colector Parabólico Compuesto (CPC) de la Universidad del Valle (Cali-Colombia).
Se empleó el diseño experimental de Taguchi (Chuen et al., 2009) para realizar los ensayos fotocatalíticos, debido a la presencia de una variable que no se puede controlar. Los factores controlables fueron el $\mathrm{pH}$ inicial de la muestra y la carga del catalizador; mientras que el factor no controlable o ruido fue la cantidad de energía UV solar acumulada en el reactor, la cual varía en el transcurso del día dependiendo de la hora y la nubosidad. De esta manera, se hizo circular por el reactor el efluente contaminante, con las respectivas condiciones de $\mathrm{pH}$ inicial y concentración de catalizador. Las pruebas se realizaron entre las 9:00 am y las 4:00 pm, con el fin de aprovechar la mayor cantidad posible de radiación solar. Con un radiómetro UV de referencia Acadus S50, se midió la energía acumulada en el reactor. En cada ensayo, se tomó una muestra al inicio del proceso, y luego una cada $6.38 \mathrm{Wh} / \mathrm{m}^{2}$ de energía UV acumulada, hasta alcanzar $38.3 \mathrm{Wh} / \mathrm{m}^{2}$. Sin embargo, para el análisis experimental de Taguchi, se tomaron en cuenta las muestras tomadas al inicio y al acumularse en el reactor $19.14 \mathrm{Wh} / \mathrm{m}^{2}$ y $38.28 \mathrm{Wh} / \mathrm{m}^{2}$. Estos valores representan la cantidad de energía en promedio que se alcanza en días nublados y soleados en el laboratorio de fotocatálisis solar de la Universidad del Valle, respectivamente (Colina, 2009).

Para establecer el efecto neto de la fotocatálisis en la mineralización de dicloxacilina, se realizaron ensayos previos para determinar el efecto de la fotólisis directa (sin catalizador) sobre el contaminante. Estas pruebas tuvieron una duración de 15 y 20 minutos, respectivamente. Se tomaron muestras al inicio y al final de cada ensayo.

Las muestras recolectadas fueron refrigeradas para su posterior análisis. Se separó el catalizador centrifugando durante 10 minutos. En cada caso, se tomaron $10 \mathrm{ml}$ de sobrenadante, se diluyeron en $100 \mathrm{ml}$ de agua del grifo y luego se midió la concentración de COT de cada solución mediante un TOC-V $\mathrm{V}_{\mathrm{CPH}}$ Shimadzu. Para evaluar el desempeño del reactor fotocatalítico CPC, se calculó el porcentaje de mineralización de 
dicloxacilina, el cual se define de acuerdo a la ecuación (8):

$$
\% \text { Mineralización }=\frac{C O T_{\mathrm{i}}-C O T_{\mathrm{f}}}{C O T_{\mathrm{i}}} \times 100
$$

En donde $C O T_{i}$ y $C O T_{f}$ representan la cantidad de carbono orgánico total al inicio y al final del ensayo de las muestras, en ppm. El porcentaje de degradación de COT fue tomado como variable de respuesta para el análisis experimental de Taguchi.

Para determinar la combinación de niveles más favorables en la degradación del contaminante considerando la variable de ruido (variación de la cantidad de energía UV acumulada), se empleó la ecuación (9):

$$
\frac{S}{R}=-10 \log \frac{1}{\mathrm{n}} \sum \frac{1}{y \mathrm{i}^{2}}
$$

En donde la razón señal-ruido $S / R$ es una función que maximiza el rendimiento del proceso, al tiempo que minimiza la pérdida esperada. En este caso, los niveles que presentaron mayor relación $S / R$, son los más favorables para la degradación de dicloxacilina y conducen a que el proceso sea menos sensible a la variación de la intensidad de radiación $U V$. Por otra parte, $\mathrm{n}$ simboliza el número de experimentos en los que interviene un nivel dado; mientras que $y_{i}$ simboliza el porcentaje de mineralización de dicloxacilina para cada nivel.

\section{Resultados y discusión}

\subsection{Ensayos de fotólisis}

La fotólisis es el proceso de descomposición de una sustancia a través de haces de luz. Al ser la fotocatálisis solar una tecnología que requiere energía lumínica, se hace necesario estimar la contribución de la fotólisis para establecer el efecto neto de la fotocatálisis. Sin embargo, el efecto aislado de la radiación solar UV sobre las muestras de dicloxacilina fue despreciable. Los porcentajes de degradación oscilaron entre $0.93 \mathrm{y}$ $1.17 \%$. Se ha reportado que la banda de absorción
UV de la dicloxacilina está entre los 225 y 274 nm (Mohamed et al., 2006). Debido a la capa de ozono, la radiación del espectro electromagnético correspondiente a estas longitudes de onda, no alcanza la superficie de la tierra. Por esta razón, la degradación de dicloxacilina por fotólisis fue insignificante.

\subsection{Ensayos de fotocatálisis}

En la Figura 4 se muestran los perfiles de degradación de dicloxacilina para los valores de $\mathrm{pH}$ y concentración de catalizador que se emplearon. Por otro lado, en la Tabla 1 se muestran los porcentajes de degradación fotocatalítica del fármaco, calculados mediante la ecuación (8).

La reducción de COT de la muestra contaminada con dicloxacilina alcanzó valores de hasta $45 \%$. Como se puede apreciar, a medida que aumenta la cantidad de energía UV acumulada en el reactor, la concentración de COT en la muestra disminuye. Esto se debe a que a mayor cantidad de energía UV, las probabilidades de generación de pares electrón-hueco en la superficie del $\mathrm{TiO}_{2}$ y de radicales $\mathrm{OH} \bullet$ son mayores (Ecs. 1-3), lo cual aumenta las reacciones de oxidación del contaminante y por lo tanto, su degradación (Ecs. 4-5).

Las Figuras 4(a), 4(b) y 4(c) muestran un comportamiento semejante respecto a la cantidad de COT eliminado. Mientras que en la Figura 4(d) se observa que la velocidad de mineralización disminuye apreciablemente después de alcanzarse $\operatorname{los} 25 \mathrm{Wh} / \mathrm{m}^{2}$ de energía UV acumulada en el reactor. A estas condiciones $(\mathrm{pH}$ inicial de $9 \mathrm{y}$ concentración de $\mathrm{TiO}_{2}$ de $\left.0.6 \mathrm{~g} / \mathrm{l}\right)$ puede haber limitaciones de adsorción física del sustrato a la superficie del catalizador debido a la carga negativa neta superficial del sólido a un $\mathrm{pH}$ por encima del punto isoeléctrico del P25.

\subsection{Efecto del pH inicial}

Las variaciones de $\mathrm{pH}$ pueden alterar la velocidad de reacción fotocatalítica, debido a que afectan la carga electrostática, el número de agregados y 
a)

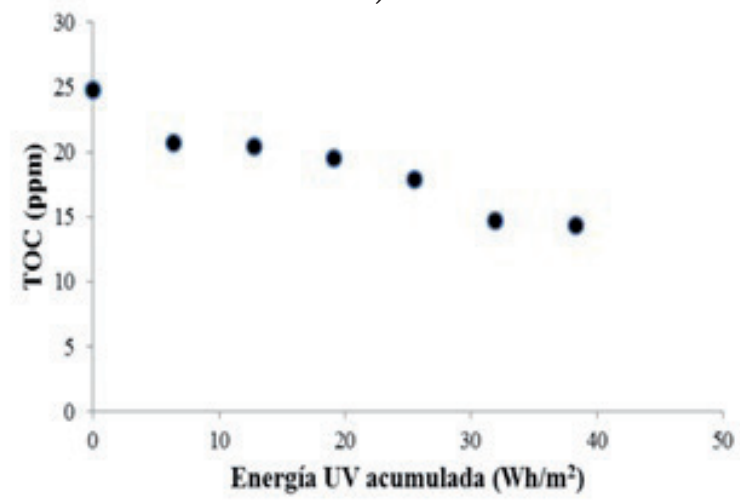

c)

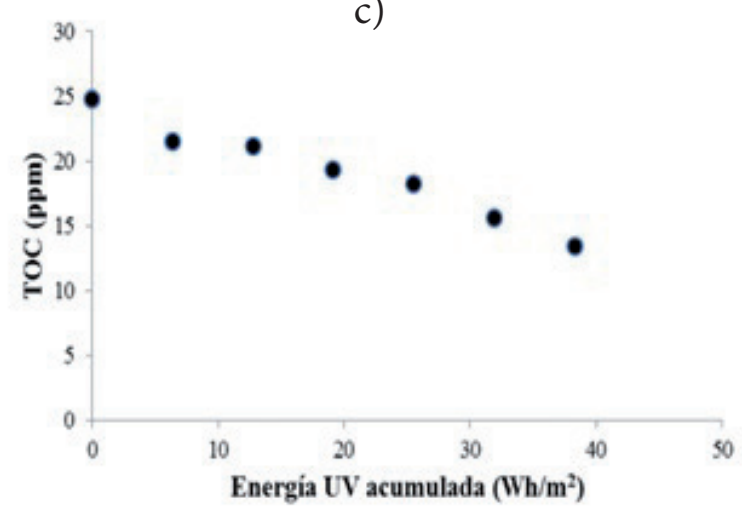

b)

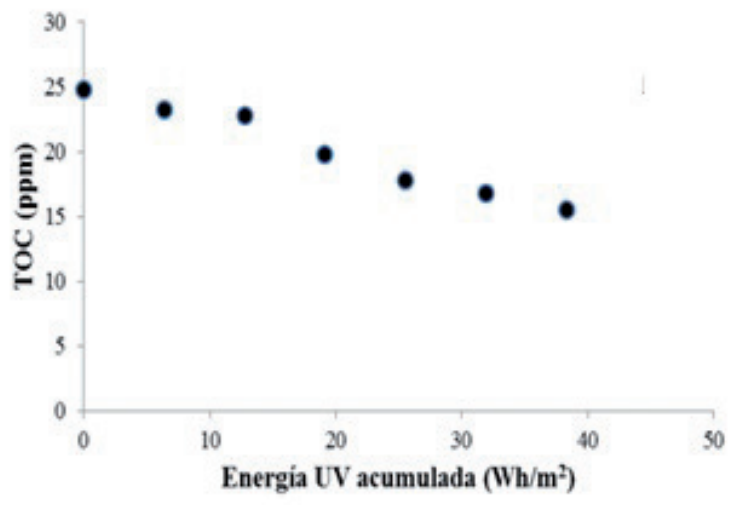

d)

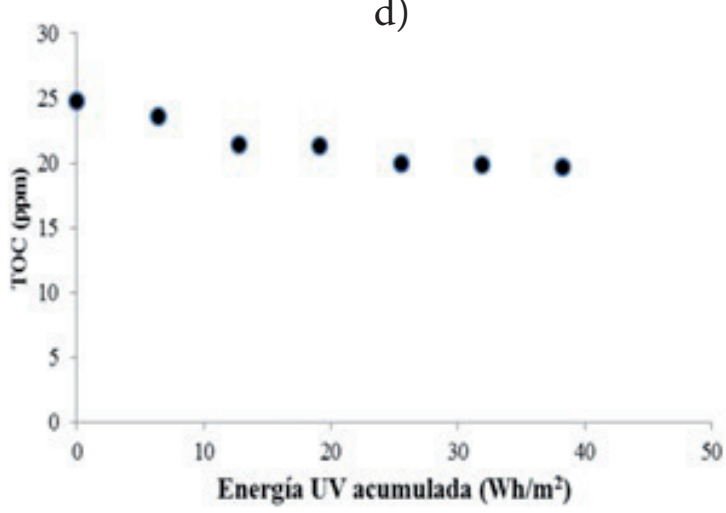

Figura 4. Perfiles de degradación de dicloxacilina en el reactor fotocatalítico a diferentes condiciones: (a) $p H=5,\left[T i O_{2}\right]=0.3$ $\mathrm{g} / \mathrm{l} ;$ (b) $\mathrm{pH}=9,\left[\mathrm{TiO}_{2}\right]=0.3 \mathrm{~g} / \mathrm{l}$; (c) $\mathrm{pH}=5,\left[\mathrm{TiO}_{2}\right]=0.6 \mathrm{~g} / \mathrm{l}$; (d) $\mathrm{pH}=9$, $\left[\mathrm{TiO}_{2}\right]=0.6 \mathrm{~g} / \mathrm{l}$.

Tabla 1. Porcentajes de mineralización de dicloxacilina en el arreglo experimental de Taguchi.

\begin{tabular}{cccc}
\hline & & \multicolumn{2}{c}{ \% Mineralización } \\
$\mathbf{p H}$ & & \multicolumn{2}{c}{ Energía UV acumulada } \\
inicial & {$\left[\mathrm{TiO}_{2}\right](\mathrm{g} / \mathrm{l})$} & $19.14 \mathrm{~W} \cdot \mathrm{h} / \mathrm{m}^{2}$ & $38.28 \mathrm{~W} \cdot \mathrm{h} / \mathrm{m}^{2}$ \\
\hline \multirow{2}{*}{5.0} & & 21.35 & 41.98 \\
& 0.3 & 22.04 & 45.82 \\
9.0 & 0.6 & 20.10 & 37.26 \\
& 0.3 & 13.89 & 20.51 \\
\hline
\end{tabular}

la posición de la banda de valencia y conducción del catalizador (Kamble et al., 2006). En la Figura 5(a) se muestran los valores de Señal-Ruido para los niveles de $\mathrm{pH}$ inicial empleados en el arreglo experimental. A un $\mathrm{pH}$ inicial de 5, la mineralización de la muestra contaminada con la dicloxacilina comercial se favoreció, esto debido a la atracción por cargas eléctricas entre las especies aniónicas del sustrato contaminante y las cargas superficiales positivas del $\mathrm{TiO}_{2} \mathrm{P} 25$, cuyo punto isoeléctrico está alrededor de un $\mathrm{pH}$ de 6.25 (Muneer et al., 2005). Por otra parte, cuando la dicloxacilina forma una solución con agua, libera iones de hidrógeno de su grupo carboxílico (ver Figura 1), lo que deja el resto de la estructura cargada negativamente. Por lo tanto, en un medio ácido, debido al aumento de la probabilidad de contacto entre el catalizador y el fármaco, la mineralización de la mezcla contaminante se ve favorecida por el incremento de la adsorción física del sustrato. 


\subsection{Efecto de la concentración del catalizador}

De acuerdo a la Figura 5(b), la degradación de dicloxacilina se favorece con una concentración de dióxido de titanio de $0.3 \mathrm{~g} / \mathrm{l}$. Una carga alta de partículas de catalizador puede aumentar inicialmente la velocidad de degradación de los contaminantes, y posteriormente, disminuirla sustancialmente por efectos de apantallamiento sobre la suspensión y dispersión de la luz (Amhed et al., 2011). A mayor concentración de partículas, hay más tendencia hacia a la aglomeración de las mismas; este fenómeno, disminuye el área superficial disponible para la absorción de fotones, lo que también afecta la eficiencia de la fotocatálisis. El número de sitios activos aumenta a mayor concentración del catalizador; pero llega un punto en donde la penetración de luz en el reactor se ve comprometida por la concentración excesiva de partículas que dispersa la energía radiante en lugar de absorberla. El equilibrio entre estos fenómenos se logra con una concentración óptima del catalizador para la reacción. Según la Figura 5(b), puede suponerse que cuando la concentración de $\mathrm{TiO}_{2}$ de $0.6 \mathrm{~g} / 1$, los efectos de aglomeración y apantallamiento se presentaron en las pruebas realizadas en el reactor. Mientras que con $0.3 \mathrm{~g} / 1$ se obtienen mejores resultados en la mineralización del contaminante, lo que es congruente con lo reportado en la literatura para este tipo de reactores (Colina-Márquez et al., 2010).

\section{Conclusiones}

La aplicación de fotocatálisis heterogénea solar con $\mathrm{TiO}_{2}$ en un reactor tipo CPC es una alternativa viable para mineralizar una muestra de agua residual contaminada con dicloxacilina comercial.

Las relaciones $\mathrm{S} / \mathrm{R}$ obtenidas a partir del diseño experimental de Taguchi, mostraron que a un $\mathrm{pH}$ inicial de 5 y 0.3 g/l de $\mathrm{TiO}_{2}$, la degradación de dicloxacilina se favorece y es independiente de las variaciones de la energía acumulada en el reactor. Con este valor de $\mathrm{pH}$, la superficie del catalizador se carga positivamente, mientras que el fármaco
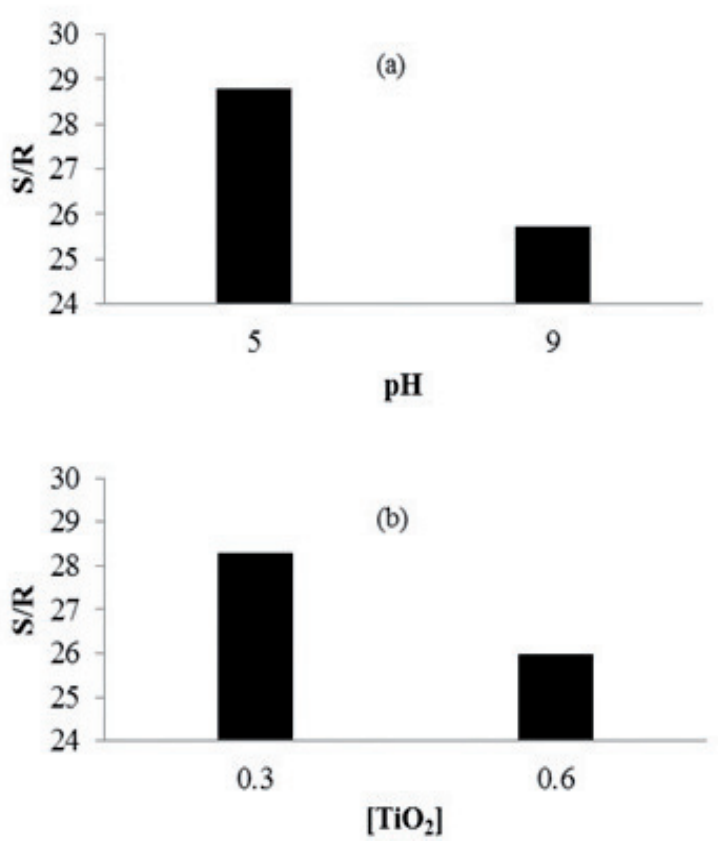

Figura 5. Señal-Ruido para el (a) pH inicial, (b) concentración del dióxido de titanio.

adquiere carga negativa al liberar hidrógenos del grupo hidroxilo cuando se disuelve en agua. Debido a esta oposición de cargas, se logra mayor adsorción del sustrato contaminante a la superficie del $\mathrm{TiO}_{2}$, lo que reduce la cantidad de COT de la muestra en solución. Por otra parte, con una concentración de $0.3 \mathrm{~g} / 1$ de $\mathrm{TiO}_{2}$, no se presentan los efectos de apantallamiento fotónico de la suspensión y la absorción de energía UV, necesaria para la generación del par electrónhueco, por parte del catalizador se favorece. Esto es congruente con lo reportado en la literatura para este tipo de reactores solares.

\section{Agradecimientos}

Los autores agradecen a los ingenieros químicos Juan Cohen Rodríguez y Leonardo Narváez Campo, por su apoyo en el montaje y realización de los ensayos experimentales; así como también al grupo GAOX de la Universidad del Valle, por facilitar el reactor fotocatalítico, y a Colciencias por los recursos financieros otorgados para el proyecto No. 110752128546 titulado “Aplicación 
de Acoples de fotocatálisis solar y tratamientos biológicos para la eliminación de contaminantes emergentes de efluentes residuales líquidos" necesarios para desarrollar esta investigación.

\section{Referencias bibliográficas}

Amhed, S., Rasul, M., Brown, R. \& Hashib, M. (2011). Influence of parameters on the heterogeneous photocatalytic degradation of pesticides and phenolic contaminants in wastewater: A short review. Journal of Environmental Management, 92(3), 311-30.

Andreozzi, R., Campanella, L., Fraysse, B., Garric, J. Gonella, A., Lo Giudice, R., Marotta, R., Pinto, G. \& Pollio, A. (2004). Effects of advanced oxidation processes (AOPs) on the toxicity of a mixture of pharmaceuticals. Water Sci. Technol., 5(5), 23-28.

Andreozzi, R., Canterino, M., Marotta, R. \& Paxeus, N. (2005). Antibiotic removal from wastewaters: the ozonation of amoxicillin. Journal of Hazardous Materials, 122(3), 243-250.

Bandala, E., Arancibia, C., Orozco, S. \& Estrada, C. (2004). Solar photoreactors comparison based on oxalic acid photocatalyticdegradation. Solar Energy, 77(5), 503-512.

Chuen, C., Sheau, L., Chung, P. \& Wen, L. (2009). The optimum conditions for preparing solid fuel briquette of rice straw by a piston-mold process using the Taguchi method. Fuel Processing Technology, 90(7-8), 1041-6.

Colina, J. (2009). Aplicabilidad de la fotocatálisis heterogénea como alternativa complementaria en el acople con un sistema biológico para la eliminación de pesticidas. Tesis Doctoral, Escuela de Ingeniería Química, Universidad del Valle, Santiago de Cali, Colombia.

Colina, J., Machuca, F. \& Li Puma, G. (2009). Photocatalytic Mineralization of Commercial Herbicides in a Pilot-Scale Solar CPC Reactor:
Photoreactor Modeling and Reaction Kinetics Constants Independent of Radiation Field. Environmental Science and Technology, 43(23), 8953-8960.

Colina-Márquez, J., Machuca-Martínez, F. \& Li Puma, G. (2010). Radiation absorption and optimization of solar photocatalytic reactors for environmental applications. Environmental Science and Technology, 44, (13), 5112-5120.

Danion, A., Disdier, J., Guillard, C., Paisse, O. \& Jaffrezic, N. (2006). Photocatalytic degradation of imidazolinone fungicide in TiO2-coated optical fiber reactor. Applied Catalysis B: Environmental, 62(3-4), 274-281.

Elmolla, E. \& Chaudhuri, M. (2010). Photocatalytic degradation of amoxicillin, ampicillin and cloxacillin antibiotics in aqueous solution using $\mathrm{UV} / \mathrm{TiO} 2$ and $\mathrm{UV} / \mathrm{H} 2 \mathrm{O} 2 / \mathrm{TiO} 2$ photocatalysis. Desalination, 252(1-3), 46-52.

Hirsch, R., Ternes, T., Haberer, K. \& Kratz, K. (1999). Occurrence of antibiotics in the aquaticenvironment. Science of The Total Environment, 225(1-2), 109-118.

Jjemba, P. (2006). Excretion and ecotoxicity of pharmaceutical and personal care products in the environment. Ecotoxicology and Environmental Safety, 63(1), 113-130.

Kamble, S., Sawant, S. \& Pangarkar, V. (2006). Photocatalytic mineralization of phenoxyacetic acid using concentrated solar radiation and titanium dioxide in slurry photoreactor. Chemical Engineering Research and Design, 84(5), 355362.

Kavitha, V. \& Palanivelu, K. (2005). Destruction of cresols by Fenton oxidation process. Water Research, 39(13), 3062-3072.

Khan, S. \& Ongerth, J. (2004). Modelling of pharmaceutical residues in Australian sewage by quantities of use and fugacity calculations. Chemosphere, 54(3), 355-367. 
Klamerth, N., Miranda, N., Malato, S., Aguera, A., Fernández, A., Maldonado, M. \& Coronado, J. (2009). Degradation of emerging contaminants at low concentrations in MWTPs effluents with mild solar photo-Fenton and TiO2. Catalysis Today, 144(1-2), 24-130.

Klauson, D., Babkina, J., Stepanova, K., Krichevskaya, M. \& Preis, S. (2010). Aqueous photocatalytic oxidation of amoxicillin. Catalysis Today, 151(1-2), 39-45.

Klavaroti, M., Mantzavinos, D. \& Kassinos, D. (2009). Removal of residual pharmaceuticals from aqueous systems by advanced oxidation processes. Environment International, 35(2), 402417.

Konstantinou, K. \& Albanis, T. (2003). Photocatalytic transformation of pesticides in aqueous titanium dioxide suspensions using artificial and solar light: intermediates and degradation pathways. Applied Catalysis B: Environmental, 42(4), 319-335.

Mascolo, G., Comparelli, R., Curri, M., Lovecchio, G., Lopez, A. \& Agostiano, A. (2007). Photocatalytic degradation of methyl red by $\mathrm{TiO} 2$ : Comparison of the efficiency of immobilized nanoparticles versus conventional suspended catalyst. Journal of Hazardous Materials, 142 (1-2), 130-137.

Méndez, F., Maldonado, M., Gimenez, J., Esplugas, S. \& Malato, S. (2009). Abatement of ibuprofen by solar photocatalysis process: Enhancement and scale up. Catalysis Today, 144(1-2), 112-116.

Mohamed, G., Nour El-Dien, F. \& Farag, U. (2006). Spectrophotometric study of the reaction mechanism between DDQ as $\pi$-acceptor and potassium iodate and flucloxacillin and dicloxacillin drugs and their determination in pure and in dosage forms. Spectrochimica Acta Part A, 65(1), 11-19.

Muneer, M., Qamar, M., Saquib, M. \& Bahnemann, D. (2005). Heterogeneous photocatalysed reaction of three selected pesticide derivatives, propham, propachlor and tebuthiuron in aqueous suspensions of titanium dioxide. Chemosphere, 61(4), 457-468.

Raj, T., Bharati, C.H., Rao, K., Rao, P., Narayan, G.K.A.S.S. \& Parikh, K. (2007). Identification and characterization of degradation products of dicloxacillin in bulk drug and pharmaceutical dosage forms. Journal of Pharmaceutical and Biomedical Analysis, 43(4), 1470-1475.

Rizzo, L., Meric, S., Guida, M., Kassinos, D. \& Belgiorno, V., (2009). Heterogenous photocatalytic degradation kinetics and detoxification of an urban wastewater treatment plant effluent contaminated with pharmaceuticals. Water Research, 43(16), 4070-4078.

Yang, L., Liu, Z., Shi, J., Zhang, Y., Hu, H. \& Shangguan, W. (2007). Degradation of indoor gaseous formaldehyde by hybrid VUV and TiO2/UV processes. Separation and Purification Technology, 54(2), 204-211.

Zhong, J., Wang, J., Gong, M., Lin, T., Liu, Z., Zhang, X. \& Chen, Y. (2007). Kinetic and degradation mechanism study on $\mathrm{Sr} 2 \mathrm{CeO} 4-$ promoted photo-oxidation of gaseous benzene. Separation and Purification Technology, 57(1), 57-62. 